\title{
CONSTRUCCIÓN DE UN ÍNDICE DE SOSTENIBILIDAD PARA LAS GANADERÍAS DE BOVINO EN CANTABRIA
}

\author{
$\underline{\text { Ibán Vázquez }}^{a *}{ }^{*}$ Elena García $^{b}$, Francisca Ruiz ${ }^{b}$, Gabriel Caymmi Vilela ${ }^{c}$, Ana Isabel García ${ }^{a}$. \\ ${ }^{a}$ Universidade de Santiago de Compostela. Escola Politécnica Superior de Enxeñaría. Dpto. Economía \\ Aplicada. (Lugo, iban.vazquez.gonzalez@usc.es) \\ ${ }^{b}$ Centro de Investigación y Formación Agrarias (CIFA). Consejería de Desarrollo Rural, Ganadería, \\ Pesca, Alimentación y Medio Ambiente del Gobierno de Cantabria \\ ${ }^{\mathrm{c}}$ Instituto Federal de Goias (Brasil)
}

\section{Resumen}

Solo una parte pequeña de los modelos utilizados para el estudio de la sostenibilidad de las ganaderías abordan las tres dimensiones: ambiental, social y económica, siendo la dimensión ambiental la que ha suscitado mayor interés en la literatura. En este trabajo proponemos construir un índice sintético de sostenibilidad, que recoja las dimensiones económica, social y ambiental, para las ganaderías cántabras. Para ello se utiliza la información obtenida de 86 entrevistas a ganaderías de vacuno de leche (64 variables) y 92 de carne (65 variables), sobre diferentes aspectos como: base territorial, ganado, producción, instalaciones y maquinaria, familia, trabajo, económicas y evolución reciente). A través de diferentes análisis factoriales de componentes principales se han construido un total de 12 indicadores.

El estudio de la sostenibilidad es relevante a la hora de fijar estrategias tanto a nivel de políticas como de gestión de las explotaciones. Los resultados permiten afirmar que, así como las ganaderías de leche muestran un mejor comportamiento en las dimensiones social y ambiental, en las ganaderías de carne es la dimensión económica la que se comporta mejor, quedando peor puntuada la dimensión social.

Palabras clave: bovino, Cantabria, sostenibilidad, análisis factorial componentes principales.

\section{Introducción y objetivos}

En las últimas décadas las ganaderías con bovino en Cantabria han experimentado un intenso proceso de ajuste y transformación (Arnalte, 2007), dando como resultado una fuerte reducción del número de explotaciones, elevación de su dimensión, intensificación, reorientación y especialización productiva (García et al., 2020). La incorporación de innovaciones tecnológicas, mejoras en procesos productivos y la reducción de los márgenes unitarios han favorecido modelos productivistas intensivos (Andrade et al., 2020). Actualmente, estos modelos comienzan a ser cuestionados por la elevada dependencia de los mercados y sus implicaciones medioambientales (TEEB, 2011).

Solo una parte pequeña de los modelos utilizados para el estudio de la sostenibilidad de las ganaderías (Van der Linden et al., 2020) abordan las tres dimensiones: ambiental, social y económica, siendo la dimensión ambiental la que ha suscitado mayor interés en la literatura (Arandia et al., 2009). Esto hace que el estudio de la sostenibilidad de las explotaciones de bovino teniendo en cuenta todas las dimensiones del concepto, se convierta en un elemento necesario a la hora de fijar estrategias tanto en el nivel de las políticas como de la propia gestión de las explotaciones, y es especialmente relevante en el actual contexto de políticas agrarias y medioambientales que se perfila en el seno de la Unión Europea (Comision europea, 2020).

El objetivo de este trabajo es medir la sostenibilidad (económica, social y ambiental) de las ganaderías con bovino en Cantabria, mediante la construcción de un índice sintético para cada una de las dimensiones.

\section{Metodología}

El material analizado procede de una encuesta realizada entre noviembre de 2016 y febrero de 2017 , mediante entrevista personal directa a 86 ganaderías de bovino de leche en Cantabria, de un total de 1.392 (FEGA, 2016). Una segunda encuesta a 92 explotaciones con vacas de carne fue llevada a cabo entre junio de 2017 y abril de 2018, de un total de 4.113 (SITRAN, 2016). La selección inicial se realizó en función de 5 estratos de producción de leche ${ }^{38}$ en la campaña 2015/2016 y de vacas de carne ${ }^{39}$ (junio 2016), mediante un muestreo aleatorio estratificado de afijación de mínima varianza de Neyman, para un error de muestreo del $5 \%$ y nivel de confianza del $95 \%$. Las preguntas se distribuyeron en seis bloques: titularidad, estructura productiva, familiar y económica, evolución reciente y perspectivas futuras.

Los índices sintéticos (IS) de sostenibilidad económica, ambiental y social se determinan en función del valor medio que presentan cuatro indicadores para cada una de las dimensiones. Los pasos para la construcción de un indicador sintético son los siguientes:

\footnotetext{
${ }^{38}$ Estrato 1: $<100 t$; estrato 2: $\geq 100 \mathrm{t} \mathrm{y}<250 \mathrm{t}$; estrato 3: $\geq 250 \mathrm{t} \mathrm{y}<500$; estrato 4: $\geq 500 \mathrm{t} \mathrm{y}<1000 \mathrm{t}$; estrato 5: $\geq 1000 \mathrm{t}$.

${ }^{39}$ Estrato 1: $<10$ vacas; estrato $2: \geq 10 \mathrm{y}<30$ vacas; estrato $3: \geq 30 \mathrm{y}<50$ vacas; estrato 4: $\geq 50 \mathrm{y}<100$ vacas; estrato 5: $\geq 100$ vacas.
} 
1-Selección de variables para confeccionar cada uno de los 12 indicadores, en función de la información disponible en las encuestas y siguiendo el marco teórico analizado (Arandia et al. 2009).

2-Realización de diversos análisis factoriales de componentes principales (AFCP), hasta hallar uno válido ${ }^{40}$.

3-Estandarización ${ }^{41}$ de las puntuaciones factoriales, en una escala de 0 a 1.

4-Cálculo de los índices de sostenibilidad (IS) para cada AFCP (Ferreira, 2021), en función del sumatorio de las puntuaciones factoriales estandarizadas multiplicado por el porcentaje unitario de la varianza.

5-Estandarización del índice de sostenibilidad (IS) para cada AFCP, en una escala de 0 a 1 y posterior transformación a escala $0-10$.

6-Interpretación del indicador de sostenibilidad (IS). Se emplea la matriz de componentes rotados para ver la relación de las variables analizadas con el propio índice; si el valor de las variables es contrario a la sostenibilidad el índice final se transforma a la inversa (10-IS).

Para la interpretación de los resultados se recurre a una tipología previa de explotaciones ${ }^{42}$. Dado que las variables seleccionadas para la construcción de los indicadores han sido diferentes para vacuno de leche y carne, principalmente debido a la información de partida, los resultados de los IS no son directamente comparables (ver variables que difieren en color negro, notas 19-30).

\section{Resultados}

En una escala de 1-10 las ganaderías de leche (Cuadro 1) obtienen 4,7 puntos en el indicador de sostenibilidad media alcanzando puntuaciones alrededor de los 5 puntos en las dimensiones social y ambiental. La dimensión económica es la peor valorada (4,1). A medida que disminuye la escala productiva lo hace también la sostenibilidad media. Esto mismo ocurre con la sostenibilidad económica y la social, ya que al aumentar la dimensión productiva aumentan los resultados económicos y los recursos para conseguir una mejora en las condiciones laborales o de bienestar animal. Sin embargo, la sostenibilidad ambiental sigue una tendencia contraria, ya que en muchos casos el aumento del tamaño trae consigo un aumento de la carga ganadera y/o una mayor necesidad de insumos, entre otros.

De los 12 índices calculados para estas ganaderías los siguientes seis indicadores superan la sostenibilidad media: autonomía económica $(7,1)$; base territorial y ganado $(6)$; instalaciones $(6,2)$; insumos $(6,6)$; titularidad $(6,0)$ y bienestar animal $(5,2)$. Por el contrario, se alcanza una puntuación muy baja $(1,2$ puntos $)$ en diversificación de actividades o en gestión ambiental. En cuanto al análisis por tipologías y respecto a la sostenibilidad económica hay que destacar que, pese a que la AP posee los mejores indicadores de rentabilidad y diversificación, en autonomía económica son superadas por las explotaciones de MP y en estructura de costes por los CS. Con relación a la sostenibilidad ambiental, las explotaciones de menor escala productiva son más sostenibles en cuanto a la base territorial y el ganado, pero no en cuanto a las instalaciones, donde son ampliamente superadas por las de mayor escala de producción. En relación con la sostenibilidad social, a excepción del índice de bienestar animal, las explotaciones de mayor escala productiva son más sostenibles.

En cuanto a las explotaciones de carne (Cuadro 2), la puntuación media obtenida es igual que en la orientación de leche $(4,7)$. Sin embargo, la diferencia entre ambas orientaciones es que, así como las de leche muestran un mejor comportamiento en las dimensiones ambiental y social que en la económica, en las explotaciones de carne es la dimensión económica $(6,0)$ la que se comporta mejor, quedando muy mal puntuada la dimensión social $(3,2)$. Al igual que en leche, a medida que disminuye la escala productiva, lo hace también la sostenibilidad media; sin embargo, en carne esto sucede para las tres dimensiones.

De los 12 índices calculados, las ganaderías de carne superan la sostenibilidad media en los cinco indicadores siguientes: rentabilidad (5,9); autonomía económica $(8,3)$; base territorial y ganado $(4,8)$; estructura de costes $(6,5)$ e insumos $(8,3)$. Por el contrario, alcanzan una puntuación muy baja en gestión ambiental $(2,3)$, condiciones laborales $(2,7)$ y relaciones territoriales (1,5). El análisis por tipología y en relación a la sostenibilidad económica, hay que subrayar que la BP superan a las AP en los indicadores de autonomía y estructura de costes. Al contrario de lo que ocurre en la orientación leche, la sostenibilidad ambiental resulta ser mayor en las AP y MP que en las BP. Por último, la sostenibilidad social es superior

\footnotetext{
${ }^{40}$ Variables no altamente correlacionadas ( R2<0,9); Análisis significativo (KMO > 0,5 y significación 0,00); Variables con una alta comunalidad (>0,5); que las variables se interpreten en la matriz de componentes rotados (sig. $>0,5)$.

41 (Valor individual - Mínimo) / (Máximo-Mínimo).

42 Bovino de leche 5 grupos: 1-Alta producción y rentabilidad (AP) (19 encuestas); 2-Media producción y rentabilidad (MP) (42 encuestas); 3-Baja producción y rentabilidad (BP) (15 encuestas); 4-Baja producción y diversificación (BPD) (7 encuestas); 5-Casos singulares (CS) (3 encuestas).

Bovino de carne 6 grupos:1- Alta producción y cebo (AP) (8 encuestas); 2-Media producción y alta rentabilidad (MPAR) (19 encuestas); 3-Media producción y baja rentabilidad (MPBR) (8 encuestas); 4-Baja producción (BP) (44 encuestas); 5-Marginales (M) (8 encuestas); 6-Casos singulares (CS) (5 encuestas.).
} 
en las explotaciones de AP. Todos los indicadores así lo atestiguan, excepto el de titularidad, donde las explotaciones de BP y M obtienen una de las mayores puntuaciones.

Cuadro 1. Índices de sostenibilidad según indicadores, dimensiones y tipologías productivas, para bovino de leche.

\begin{tabular}{|c|c|c|c|c|c|c|c|}
\hline \multicolumn{3}{|c|}{ TIPOLOGÍA PRODUCTIVA Y SOCIOECONÓMICA AP } & \multirow{2}{*}{$\frac{\mathbf{M P}}{4,3 \mathrm{~b}}$} & \multirow{2}{*}{$\frac{\mathbf{B P}}{3,6 \mathrm{~b}}$} & \multirow{2}{*}{$\frac{\text { BPD }}{4,7 a b}$} & \multirow{2}{*}{$\frac{\text { CS }}{5,1 \mathrm{ab}}$} & \multirow{2}{*}{$\frac{\text { Total }^{43}}{4,6^{* * *}}$} \\
\hline SOSTENIBILIDAD & Rentabilidad $^{44}$ & $6,0 \mathrm{a}$ & & & & & \\
\hline \multirow[t]{3}{*}{ ECONÓMICA } & Autonomía ${ }^{45}$ & $6,6 a b$ & $7,9 \mathrm{a}$ & $6,2 \mathrm{ab}$ & $6,4 \mathrm{ab}$ & $3,9 b$ & $7,1 * * *$ \\
\hline & Diversificación $^{46}$ & 1,8 & 0,9 & 1,6 & 0,5 & 0,6 & $1,2 \mathrm{~ns}$ \\
\hline & Estructura de costes ${ }^{47}$ & $4,8 \mathrm{a}$ & $2,8 b$ & $4,1 \mathrm{ab}$ & $3,2 \mathrm{ab}$ & $6,7 \mathrm{a}$ & $3,6 * * *$ \\
\hline SOSTENIBILIDAD & Base territorial y ganado ${ }^{48}$ & $3,5 \mathrm{c}$ & $6,2 \mathrm{~b}$ & $8,0 \mathrm{a}$ & $6,5 \mathrm{ab}$ & 7,3ab & $6,0 * * *$ \\
\hline \multirow[t]{3}{*}{ AMBIENTAL } & Instalaciones ${ }^{49}$ & $7,4 \mathrm{a}$ & $6,4 \mathrm{a}$ & $5,2 \mathrm{~b}$ & $4,2 \mathrm{~b}$ & $6,2 \mathrm{ab}$ & $6,2 * * *$ \\
\hline & Insumos $^{50}$ & $3,8 b$ & $7,1 \mathrm{a}$ & $7,6 \mathrm{a}$ & $8,6 \mathrm{a}$ & $6,5 \mathrm{ab}$ & $6,6^{* * *}$ \\
\hline & Gestión global $^{51}$ & 1,2 & 1,2 & 1,3 & 0,7 & 1,6 & $1,2 \mathrm{~ns}$ \\
\hline SOSTENIBILIDAD & Titularidad $^{52}$ & $7,4 a$ & $5,9 \mathrm{ab}$ & $4,4 \mathrm{~b}$ & $5,2 \mathrm{ab}$ & $7,5 \mathrm{a}$ & $6,0^{*}$ \\
\hline \multirow[t]{3}{*}{ SOCIAL } & Condiciones laborales $^{53}$ & $6,6 a$ & $3,9 \mathrm{~b}$ & $3,0 \mathrm{bc}$ & $1,5 \mathrm{c}$ & $4,0 \mathrm{~b}$ & $4,1 * * *$ \\
\hline & Relaciones territoriales ${ }^{54}$ & $6,3 \mathrm{a}$ & $4,4 b$ & $1,9 \mathrm{c}$ & $1,1 \mathrm{c}$ & $4,4 b$ & $4,1 * * *$ \\
\hline & Bienestar animal $^{55}$ & 5,6 & 4,8 & 5,5 & 5,3 & 6,9 & $5,2 \mathrm{~ns}$ \\
\hline \multirow{3}{*}{\multicolumn{2}{|c|}{$\begin{array}{l}\text { Sostenibilidad económica } \\
\text { Sostenibilidad ambiental } \\
\text { Sostenibilidad social }\end{array}$}} & $4,8 \mathrm{a}$ & $4,0 \mathrm{~b}$ & $3,8 \mathrm{~b}$ & $3,7 \mathrm{~b}$ & $4,1 \mathrm{~b}$ & $4,1 *$ \\
\hline & & $4,0 \mathrm{~b}$ & $5,2 \mathrm{a}$ & $5,5 \mathrm{a}$ & $5,0 \mathrm{ab}$ & $5,4 \mathrm{a}$ & $5,0 * * *$ \\
\hline & & $6,5 \mathrm{a}$ & $4,7 \mathrm{~b}$ & $3,7 \mathrm{c}$ & $3,3 \mathrm{c}$ & $5,7 \mathrm{ab}$ & $4,9 * * *$ \\
\hline \multicolumn{2}{|c|}{ SOSTENIBILIDAD MEDIA } & $5,1 \mathrm{a}$ & $4,6 \mathrm{ab}$ & $4,4 \mathrm{~b}$ & $4,0 \mathrm{~b}$ & $5,1 \mathrm{a}$ & $4,7 * * *$ \\
\hline
\end{tabular}

Cuadro 2. Índices de sostenibilidad según indicadores, dimensiones y tipologías productivas, para vacuno de carne.

\begin{tabular}{|c|c|c|c|c|c|c|c|c|}
\hline TIPOLOGÍA PRODUC & TIVA Y SOCIOECONÓMICA & $\mathbf{A P}$ & MPAR & MPBR & BP & $\mathbf{M}$ & CS & Total $^{6}$ \\
\hline \multirow{5}{*}{$\begin{array}{l}\text { SOSTENIBILIDAD } \\
\text { ECONÓMICA }\end{array}$} & Rentabilidad $^{56}$ & $7,3 \mathrm{a}$ & $6,3 \mathrm{ab}$ & $5,9 b$ & $5,9 b$ & $4,1 \mathrm{c}$ & $4,7 \mathrm{bc}$ & $5,9 * * *$ \\
\hline & Autonomía $^{57}$ & 7,8 & 8,9 & 8,7 & 8 & 8,4 & 9,1 & $8,3 \mathrm{~ns}$ \\
\hline & Diversificación $^{58}$ & $6,2 \mathrm{a}$ & $2,9 b$ & $2,8 b$ & $3,6 b$ & $2,1 b$ & $2,9 b$ & $3,4 * *$ \\
\hline & Estructura de costes ${ }^{59}$ & $6,6 \mathrm{a}$ & $6,6 \mathrm{a}$ & $4,3 b$ & $7,1 \mathrm{a}$ & $5,4 \mathrm{ab}$ & $6,3 \mathrm{ab}$ & $6,5 * * *$ \\
\hline & Base territorial y ganado ${ }^{60}$ & $4,8 \mathrm{ab}$ & $6,5 \mathrm{a}$ & $4,4 \mathrm{ab}$ & $4,4 \mathrm{~b}$ & $3,6 b$ & $4,2 \mathrm{ab}$ & $4,8 * * *$ \\
\hline \multicolumn{9}{|c|}{$\begin{array}{l}{ }^{43} \text { Media simple sobre el valor medio de cada indicador. Significación estadística: *** } 0,1 \% \text {; **1 \%; *5\%; t Tendencia }(5-10 \%) \text {; } \\
\text { ns } \geq 10 \% \text {. Subíndices con distinta letra indican subconjuntos homogéneos significativamente diferentes al nivel del } 5 \% \text {. } \\
{ }^{44} \mathrm{MN} \text { total }(€) ; \mathrm{MN} / \mathrm{UTA}(€) ; \mathrm{MN} / 1.0001(€) ; \mathrm{PB} / \mathrm{CT} ; \mathrm{B} / \mathrm{PB} \text {. }\end{array}$} \\
\hline \multicolumn{9}{|c|}{$\begin{array}{l}45 \text { Subvenciones/PB }(\%) \text {; Préstamos pendientes }(€) \text {; Coste arrendamiento (€/año); Incremento contratación servicios externos } \\
(\mathrm{Si} / \mathrm{No}) \text {; Factores Externos/CT }(\%) \text {; Externalización actividades (SI/No). }\end{array}$} \\
\hline \multicolumn{9}{|c|}{$\begin{array}{l}{ }^{46} \text { Otros ingresos agrarios } / \mathrm{PB}(\%) \text {; OAL }(€) \text {; Cebo de vacas de desvieje }(\mathrm{Si} / \mathrm{No}) \text {; Cebo de terneros (SI/No); Transformación } \\
\text { productos explotación }(\mathrm{Si} / \mathrm{No}) \text {; Diversificación reciente }\left(\mathrm{N}^{\circ}\right) \text {. }\end{array}$} \\
\hline \multicolumn{9}{|c|}{$\begin{array}{l}{ }^{47} \text { Coste alimentación comprada/CT }(\%) \text {; Costes específicos/CT }(\%) ; \text { Coste de oportunidad/PB (\%); Factores externos/CT }(\%) \text {; } \\
\text { CT/PB (Ratio); Reducción costes producción (Si/No). }\end{array}$} \\
\hline \multicolumn{9}{|c|}{$\begin{array}{l}{ }^{48} \text { Carga ganadera (Vacas/haSAU); SAU pastos/SAU total (\%); Consumo concentrado (kg/vaca/día); Longevidad (Lactaciones); } \\
\text { Mayoría fincas }-3 \mathrm{~km}(\mathrm{Si} / \mathrm{No}) \text {. }\end{array}$} \\
\hline \multicolumn{9}{|c|}{$\begin{array}{l}{ }^{49} \text { Máximo almacenaje fosa (meses); Construcción establo reciente (año); Potencia tractor más potente (cv); Medidas de eficiencia } \\
\text { energética }\left(\mathrm{n}^{\circ}\right) \text {; Fosa purín cubierta (Si/No); Superficie establo por vaca (m2). }\end{array}$} \\
\hline \multicolumn{9}{|c|}{$\begin{array}{l}50 \text { Abono químico (kg/ha SAU); Coste fitosanitarios (€/haSAU); Coste carburantes y lubricantes (€/haSAU); Coste agua, luz, } \\
\text { teléfono (€/haSAU). }\end{array}$} \\
\hline \multicolumn{9}{|c|}{$\begin{array}{l}51 \text { Superficie interés ecológico (Si/No); Trabajos mantenimiento pastos (Si/No); Estrategia extensificación (Si/No); Introducción } \\
\text { nuevos cultivos ( } \mathrm{Si} / \mathrm{No}) \text {. }\end{array}$} \\
\hline \multicolumn{9}{|c|}{52 Edad (años); Nivel de estudios (4 niveles); Continuidad futura (Si/No); Sociedades familiares (Si/No). } \\
\hline \multicolumn{9}{|c|}{53 Días libres al año (días); Contrato de servicios agrarios (Si/No); Limpieza automática (Si/No); Sistema de ordeño (5 tipos). } \\
\hline \multicolumn{9}{|c|}{$\begin{array}{l}54 \text { Integración cooperativa (Si/No); Aumento servicios agrarios (Si/No); Aumento mano de obra (Si/No); Leche más calidad } \\
\text { (Si/No); Certificación de calidad (Si/No); Control lechero (Si/No). }\end{array}$} \\
\hline \multirow{2}{*}{\multicolumn{9}{|c|}{$\begin{array}{l}55 \text { Ventilación ( } \mathrm{n}^{\mathrm{o}} \text { sistemas); Cepillos (Si/No); Arrobaderas (Si/No); Cornadizas/cama }\left(\mathrm{N}^{\mathrm{o}}\right) \text {; Superficie/vaca (m2); Tipo cama establo } \\
(3 \text { niveles); Duración pastoreo (meses); Duración pastoreo diario (horas). } \\
56 \text { MN total (€); MN/UTA }(€) ; \text { MN/vaca (€); PB/CT; B/PB. }\end{array}$}} \\
\hline & & & & & & & & \\
\hline \multirow{2}{*}{\multicolumn{9}{|c|}{$\begin{array}{l}57 \text { Subvenciones/PB (\%); Préstamos pendientes }(€) \text {; Coste arrendamiento (€/año); Incremento factores externos (Si/No); Factores } \\
\text { Externos/CT }(\%) \text {; aumento contratación servicios agrarios (SI/No). }\end{array}$}} \\
\hline & & & & & & & & \\
\hline \multicolumn{9}{|c|}{$\begin{array}{l}58 \text { Otros ingresos agrarios /PB (\%); OAL (€); Recría novillas venta (Si/No); Cebo de terneros (SI/No); Diversificación reciente } \\
\left(\mathrm{N}^{\mathrm{o}}\right) \text {. }\end{array}$} \\
\hline \multicolumn{9}{|c|}{$\begin{array}{l}{ }^{59} \text { Coste alimentación comprada/CT (\%); Costes específicos/CT (\%); Coste de oportunidad/PB (\%); CT/PB (Ratio); } \\
\text { Amortizaciones/PB (\%). }\end{array}$} \\
\hline
\end{tabular}




\begin{tabular}{|c|c|c|c|c|c|c|c|c|}
\hline \multirow{3}{*}{$\begin{array}{l}\text { SOSTENIBILIDAD } \\
\text { AMBIENTAL }\end{array}$} & Instalaciones $^{61}$ & 6,1 & 4,8 & 4,4 & 4,4 & 4,7 & 6,5 & $4,7 \mathrm{~ns}$ \\
\hline & Insumos $^{62}$ & 8,3 & 7,8 & 9,5 & 8,3 & 8,1 & 8,3 & $8,3 \mathrm{~ns}$ \\
\hline & Gestión global $^{63}$ & 1,9 & 3,3 & 2,3 & 2,1 & 1,7 & 1,7 & $2,3 \mathrm{~ns}$ \\
\hline \multirow{4}{*}{$\begin{array}{l}\text { SOSTENIBILIDAD } \\
\text { SOCIAL }\end{array}$} & Titularidad $^{64}$ & 4,5 & 3,8 & 6,0 & 4,7 & 5,3 & 4,4 & $4,7 \mathrm{~ns}$ \\
\hline & Condiciones laborales ${ }^{65}$ & $4,6 \mathrm{a}$ & $3,1 \mathrm{ab}$ & $2,1 b$ & $2,3 b$ & $2,1 b$ & $3,6 \mathrm{ab}$ & $2,7 * *$ \\
\hline & Relaciones territoriales ${ }^{66}$ & 2,8 & 0,3 & 1,1 & 1,9 & 1,8 & 0,6 & $1,5 \mathrm{~ns}$ \\
\hline & Bienestar animal $^{67}$ & $3,8 \mathrm{ab}$ & $5,7 \mathrm{a}$ & $2,9 b$ & $3,5 \mathrm{~b}$ & $2,8 b$ & $2,6 \mathrm{~b}$ & $3,8 * * *$ \\
\hline \multicolumn{2}{|c|}{ Sostenibilidad económica } & $7,0 \mathrm{a}$ & $6,2 \mathrm{ab}$ & $5,4 \mathrm{bc}$ & $6,1 \mathrm{ab}$ & $5,0 \mathrm{c}$ & $5,8 \mathrm{~b}$ & $6,0 * * *$ \\
\hline \multicolumn{2}{|c|}{ Sostenibilidad ambiental } & $5,3 \mathrm{ab}$ & $5,6 \mathrm{a}$ & $5,1 \mathrm{ab}$ & $4,8 b$ & $4,5 b$ & $5,2 \mathrm{ab}$ & $5,0 *$ \\
\hline \multicolumn{2}{|c|}{ Sostenibilidad social } & 3,9 & 3,2 & 3,0 & 3,1 & 3,0 & 2,8 & $3,2 \mathrm{~ns}$ \\
\hline \multicolumn{2}{|c|}{ SOSTENIBILIDAD MEDIA } & $5,4 a$ & $5,0 \mathrm{ab}$ & $4,5 \mathrm{bc}$ & $4,7 \mathrm{~b}$ & $4,2 \mathrm{c}$ & $4,6 b c$ & $4,7 * *$ \\
\hline
\end{tabular}

\section{Conclusiones}

A pesar de que los índices de sostenibilidad calculados no son directamente comparables para las dos orientaciones, concluimos que la sostenibilidad media está próxima al aprobado, con un mayor desempeño de la dimensión ambiental y social en las de leche y en la económica y ambiental para las de carne.

Las características productivas y socioeconómicas de las ganaderías determinan su sostenibilidad. Con la única excepción de la dimensión ambiental en vacuno de leche, cuanto mayor es la dimensión productiva mayor es el índice de sostenibilidad por la influencia de los indicadores sociales y económicos.

\section{Bibliografía}

Andrade, D., Pasini, F., Scarano, F. R. (2020). "Syntropy and innovation in agricultura". Current Opinion in Environmental Sustainability, 45:20-24.

Arandia, A., Intxaurrandieta, J. M., Santamaria, P., Del Hierro, O., Nafarrate, L., Icaran, C., Lopez, E., Pinto, M. y Mangado, J. M. (2009). Development of a tool to diagnose economic, environmental and social sustainability of animal husbandry systems. Application to dairy farming. Sociedad Española para el Estudio de los Pastos (SEEP). Huesca.

Arnalte, E. (2007). Economía política del proceso de ajuste estructural en la agricultura de los países desarrollados. (Ed.): Políticas agrarias y ajuste estructural en la agricultura española (pp.17-54). Madrid: MAPA.

Comision Europea. (2020). "Comunicacion de la comision al parlamento europeo, al consejo europeo, al consejo, al comite economico y social europeo y al comite de las regiones". El Pacto Verde Europeo. Bruselas, $\operatorname{COM(2019)} 640$ final. https://eur-lex.europa.eu/legalcontent/ES/TXT/?qid=1596443911913\&uri=CELEX:52019DC0640\#document2.

FEGA (2016). Fondo español de garantía agraria. MAPA [Consultado 29 de junio de 2016].

García, E., Ruiz, F., García, A.I. y Vázquez, I. (2020). “Caracterización productiva y socioeconómica de las explotaciones con vacas en Cantabria". Revista de Estudios Agrosociales y Pesqueros, 256:175-196.

SITRAN (2016). Ministerio de Agricultura, Pesca y Alimentación. [Consultado 29 de junio de 2016].

TEEB (Ed.) 2011. The economics of ecosystems and biodiversity for national and international policy makers. London: Routlidge.

Van der Linden, A., de Olde, E.M., Mostert, P.R. y de Boer, I.J.M. (2020). "A review of European models to assess the sustainability performance of livestock production systems". Agricultural Systems, 182:1028-1042.

\footnotetext{
${ }^{61}$ Fosa purin (Si/No); Construcción establo reciente (año); Potencia tractor más potente (cv); Distancia establo vivienda (m); tractores $\left(n^{o}\right)$; cornadizas/vaca $\left(n^{o}\right)$; potencia total tractores (cv/haSAU); posibilidad ampliar establo (Si/No).

${ }^{62}$ Coste abono químico (€/ha SAU); Coste fitosanitarios (€/haSAU); Coste plásticos (€/haSAU); Coste carburantes y lubricantes $(€ /$ haSAU).

${ }^{63}$ Estrategia extensificación ( $\left.\mathrm{Si} / \mathrm{No}\right)$; presencia razas autóctonas (Si/No); diversificación cultivos (Si/No); aprovechamiento pastos comunales $(\mathrm{Si} / \mathrm{No})$.

${ }^{64}$ Edad (años); Nivel de estudios (4 niveles); Continuidad futura (Si/No); Sociedades familiares (Si/No); Titularidad mujer (Si/No); Ocupación tiempo completo titular (Si/No).

${ }^{65}$ Días libres al año (días); Contrato de servicios agrarios ( $\left.\mathrm{Si} / \mathrm{No}\right)$; Tractores ( ${ }^{\mathbf{o}}$ ); Potencia tractor más potentes (cv).

${ }^{66}$ Contratación servicios agrarios (Si/No); Aumento mano de obra (Si/No); Certificación de calidad (Si/No); Transf. productos explotación (Si/No); Tursimo y energía (Si/No); actividades exteriores $\left(\mathbf{n}^{\circ}\right)$.

${ }^{67}$ Cornadizas/cama $\left(\mathrm{N}^{\circ}\right)$; Longevidad (años); Duración estabulación invernal (días); Pastos comunales (Si/No); Carga ganadera (Vacas/ha SAU), Vacas muertas (\%).
} 